\title{
The Physical Properties of Red Supergiants: Comparing Theory and Observations
}

\author{
Philip Massey ${ }^{1}$, Emily M. Levesque ${ }^{2}$, Bertrand Plez $^{3}$ \\ and Knut A. G. Olsen ${ }^{4}$ \\ ${ }^{1}$ Lowell Observatory, 1400 W. Mars Hill Rd., Flagstaff, AZ 86001, USA \\ email: Phil.Massey@lowell.edu \\ ${ }^{2}$ Institute for Astronomy, University of Hawaii, \\ 2680 Woodlawn Drive, Honolulu, HI 96822, USA \\ email: emsque@ifa.hawaii.edu \\ ${ }^{3}$ GRAAL, Université Montpellier II, CNRS, 34095 Montpellier, France \\ email: Bertrand.Plez@graal.univ-montp.fr \\ ${ }^{4}$ Gemini Science Center, NOAO, P.O. Box 26732, Tucson, AZ 85726-6732, USA \\ email: kolsen@noao.edu
}

\begin{abstract}
Red supergiants (RSGs) are an evolved stage in the life of intermediate massive stars $\left(\leqslant 25 \mathrm{M}_{\odot}\right)$. For many years, their location in the H-R diagram was at variance with the evolutionary models. Using the MARCS stellar atmospheres, we have determined new effective temperatures and bolometric luminosities for RSGs in the Milky Way, LMC, and SMC, and our work has resulted in much better agreement with the evolutionary models. We have also found evidence of significant visual extinction due to circumstellar dust. Although in the Milky Way the RSGs contribute only a small fraction $(<1 \%)$ of the dust to the interstellar medium (ISM), in starburst galaxies or galaxies at large look-back times, we expect that RSGs may be the main dust source. We are in the process of extending this work now to RSGs of higher and lower metallicities using the galaxies M31 and WLM.
\end{abstract}

Keywords. stars: atmospheres - circumstellar matter - stars: evolution - stars: late-type stars: mass loss - supergiants

\section{Introduction}

Those of us here who have worked on massive stars for a while are probably all attracted by stellar physics at the extremes. For O-type stars, we are dealing with stars that are as massive and luminous as stars come, and as hot as main-sequence stars can get. To properly assess their physically properties through spectroscopic analysis has required not only the introduction of non-LTE atmosphere models (Mihalas \& Auer 1970, Auer \& Mihalas 1972) but an additional thirty years of developments, such as the inclusion of mass loss (Abbott \& Hummer 1972, Kudritzki 1976), the inclusion of hydrodynamics of the stellar wind (Gabler et al. 1989; Kudritzki \& Hummer 1990; Puls et al. 1996), and, most recently, the full inclusion of line blanketing (Hillier \& Miller 1989; Hillier et al. 2003; Herrero, Puls, \& Najarro 2002). (For a recent summary, see Massey et al. 2004 and Massey et al. 2005a). The study of LBVs and WRs is equally exciting, stars where radiation pressure dances with gravity to see who will lead (Lamers 1997, Smith \& Owocki 2006), and where high mass loss rates are continuous rather than episodic due to high metal content in the stellar atmosphere (Crowther 2007 and references therein).

However, largely ignored until now are the red supergiants (RSGs). The physical conditions in these stars are, in their own way, equally extreme. They have the largest physical sizes of any stars (up to $1500 \times$ the radius of the sun; see Levesque et al. 2005, 
here after Paper I). This large physical size invalidates the usual assumptions of plane parallel geometry. The velocities of the convective layers in these stars' atmospheres are supersonic, giving rise to shocks (Freytag, Steffen, \& Dorch 2002), and making the stars' photospheres very asymmetric and invalidating mixing-length assumptions. Their extremely cool temperatures $(3400-4300 \mathrm{~K})$ lead to the the presence of molecules in their atmospheres, requiring the inclusion of extensive molecular opacity sources in any realistic model atmosphere. From an observational point of view, the large (negative) bolometric corrections and their sensitivity to the adopted temperature complicate the transformation from the observed color-magnitude diagram to the physical H-R diagram in much the same way as it does for the O-type stars.

Recent advances in stellar atmosphere models for cool stars (e.g., Plez 2003) have allowed the first reasonable determination of the physical properties of these stars, in much the same way that the non-LTE H and He models of Auer \& Mihalas (1972) allowed the first reasonable determination of the physical properties of O-type stars by Conti (1973). And while we may still have a way to go, we believe our answers will hold up as well as those that Conti (1973) have, which is really pretty good (see Massey et al. 2005a).

I find it personally interesting that there has been a real aversion to looking at what happens to a massive star as it heads over to the far right side of the H-R diagram. I think this is cultural - for many years much of the "massive star community" really thought of itself as the "hot star community", with the exception of a few workers, most notably our good colleague Roberta Humphreys, whose early work on supergiants in the Milky Way and other Local Group galaxies (such as Humphreys 1978, 1979a, 1979b, 1980a, 1980b, 1980c, Humphreys \& Davidson 1979, Humphreys \& Sandage 1980) certainly spurned my own interest in the field, and whose presence at these symposia always reminds us that there's more to the life of a massive star than the $\mathrm{O}$ and WR stages.

Let us briefly review what the evolutionary tracks predict RSGs come from. In Fig. 1 we show the tracks covering a range of a factor of 10 in metallicity, from $z=0.004$ (SMClike) to $\mathrm{z}=0.020$ (solar) to $\mathrm{z}=0.040$ (M31-like). I have drawn a vertical line at an effective temperature of $4300 \mathrm{~K}$, which roughly corresponds to that of a K0 I. Stars to the right of that line we are calling RSGs. At solar metallicities we expect that stars with initial masses $\leqslant 25 \mathrm{M}_{\odot}$ will become RSGs. At lower metallicities (SMC-like) the upper mass limit for RSGs is probably a bit higher-maybe $30 \mathrm{M}_{\odot}$ ? - it's hard to tell because of the quantization of the tracks. The upper mass tracks go much further to the right at this low metallicity, but stop short of the RSG dividing lines - these $30-60 \mathrm{M}_{\odot}$ stars become F- and G-type supergiants, but not K or M. At higher metallicity (M31) the limit is definitely lower, around $20 \mathrm{M}_{\odot}$. In the case of solar metallicity the $25 \mathrm{M}_{\odot}$ track turns back to the blue, and in fact such a star should become a WR after the RSG phase.

One more thing of note is that the tracks don't extend very far to the right of the K0 I (vertical) line in the SMC - the RSGs in the SMC shouldn't be very late, mostly K through M0, say. At higher metallicities they extend further to cooler effective temperatures. This is consistent with the change in the average RSG type observed in the SMC, LMC, and Milky Way (Elias, Frogel, \& Humphreys 1985, Massey \& Olsen 2003).

That said, when we began worrying about the issue of the physical properties of RSGs it was because if one placed the "observed" location of RSGs on the H-R diagram, they missed the tracks entirely: the alleged effective temperatures and luminosities were cooler and higher than those predicted by the evolutionary tracks. This was first noticed by Massey \& Olsen (2003) for the SMC and LMC, but we quickly confirmed that the problem also existed for the Milky Way sample (Massey 2003a). When I mentioned this issue at the Lanzarote meeting (Massey 2003b) Daniel Schaerer came up to me afterwards and said 

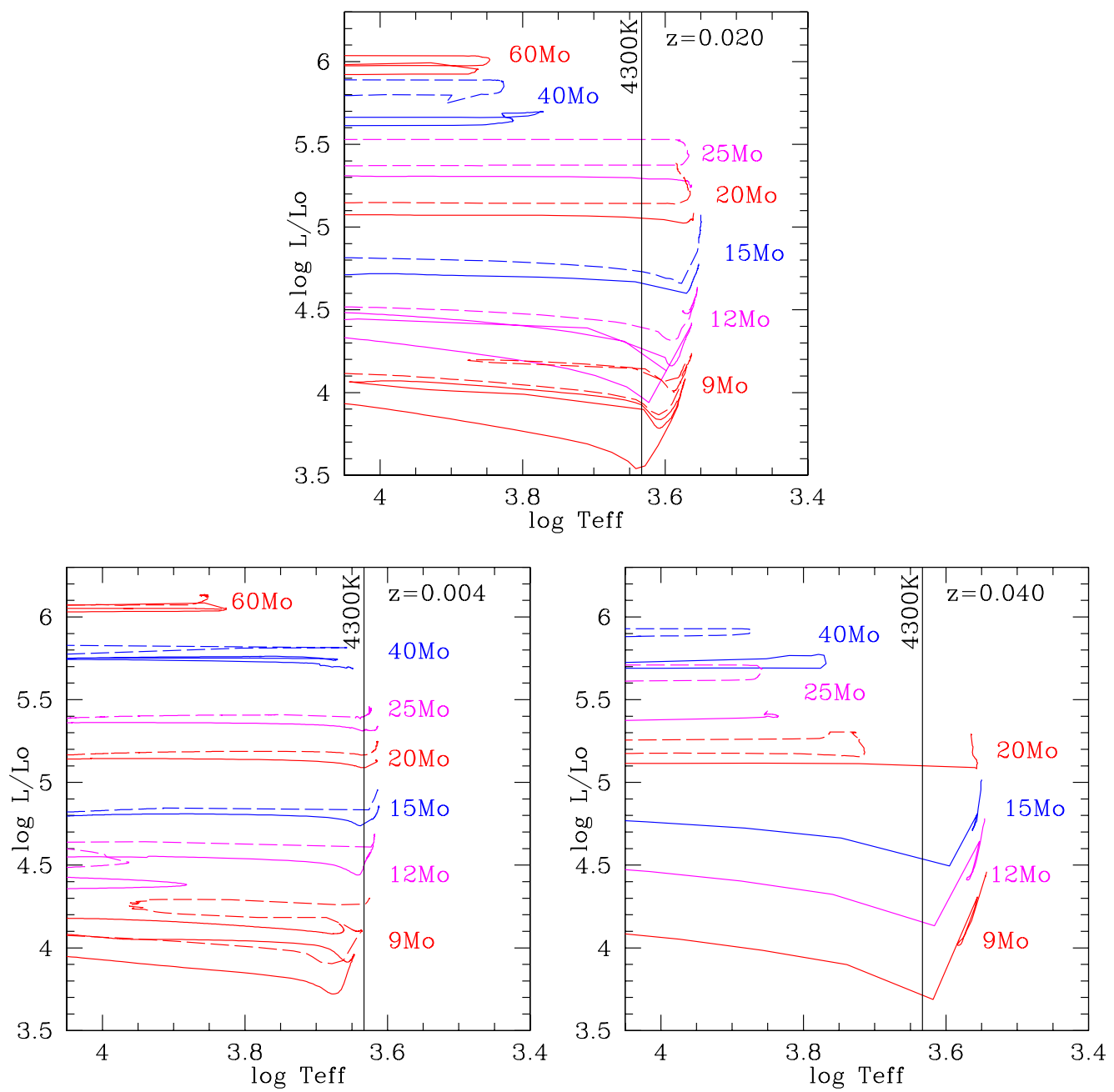

Figure 1. Effects of metallicity on the evolutionary tracks in the RSG region. The tracks for $\mathrm{z}=0.020$ (solar) are from Meynet \& Maeder (2003), for $\mathrm{z}=0.004$ (SMC-like) are from Meynet \& Maeder (2000), and for $\mathrm{z}=0.040$ (M31-like) are from Meynet \& Maeder (2005) and Meynet et al. 1994). Solid curves denote the tracks with no initial rotation, while the dashed lines correspond to initial rotations of $300 \mathrm{~km} \mathrm{~s}^{-1}$. The black vertical line marks a temperature of $4300 \mathrm{~K}$, roughly that of a K0 I at both solar and SMC metallicity (Papers I, II).

really, this was not a problem, since how far to the right the tracks went were heavily dependent upon such issues as how the mixing length was treated (see, for example, Maeder \& Meynet 1987). However, this did not explain the issue of the luminosities being too high, and as an observer I was more concerned with what if the "observations" were wrong? Because, of course we don't "observe" effective temperatures and bolometric luminosities; instead, we obtain photometry and spectroscopy and use some relationship to convert these to physical properties. Indeed, further reading convinced me that there could be a serious problems, as much of what we "knew" about the effective temperature scale of RSGs were derived from lunar occultations of red giants (not supergiants); see discussion in Massey \& Olsen (2003). 
What would be involved in determining the effective temperatures of RSGs "right"? We really need models that have enough physics in them to correctly reproduce temperaturesensitive spectral features. The participants at this conference (mostly) understand what was involved in getting there for O-type stars. RSGs present their own challenges, as noted above. Fortunately, at the time I got intrigued by this problem, sophisticated models that were up to the task were becoming available. A modern version of these MARCS models was described by Plez, Brett, \& Nordlund (1992), based upon the earlier work of Gustafsson et al. (1975). These are static, LTE, opacity-sampled models, and the current version (Plez 2003; Gustafsson et al. 2003) includes improved atomic and molecular opacities and sphericity.

\section{RSGs in the Milky Way}

In Paper I we obtained moderate-resolution spectrophotometry of 74 Galactic RSGs, which we then compared to the models. Our primary selection criterion was that the RSG had to be in a cluster or an association with a relatively well determined distance from the OB stars (Humphreys 1978, Garmany \& Stencil 1992). We used a grid of models with effective temperatures $3000-4500 \mathrm{~K}$ in increments of $25 \mathrm{~K}$, and $\log g=-1$ to +1 [cgs] in steps of $0.5 \mathrm{deg}$. We would typically begin by reddening the $\log g=0.0$ model spectra of various effective temperatures by different amounts (using a Cardeli, Clayton, \& Mathis 1989 reddening law with $R_{V}=3.1$ ) until we got a good match to the depths of the molecular bands (principally $\mathrm{TiO}$ ) and continuum shape of the spectra of the star. We would then see if the derived luminosity implied a surface gravity consistent with the $\log g=0.0$; if not, we used a more appropriate model. In practice, the value we determined for the effective temperatures did not depend upon our 0.5 dex uncertainty in the adopted surface gravity, and the $A_{V}$ was affected by $<0.3$ mag.

When all was said and done, we had derived a new effective temperature scale which was significantly warmer than the older ones. It is shown by the points in Fig. 2. Their error bars reflect the standard deviation of the mean of our determinations; for the M stars (where we can use the TiO bands) our precision was $\sim 25 \mathrm{~K}$, or about $0.7 \%$ compare this to the typical $2000 \mathrm{~K}(5 \%)$ uncertainty we have when fitting O stars and their weak lines!

What did that do to the placement of stars in the H-R diagram? Just what we hoped! We show the situation (old and new) in Fig. 3. Now there is excellent agreement both in the effective temperatures, and in the upper luminosities, of RSGs in the Milky Way.

One of the cute things to come out of Paper I is the answer to "How large do normal stars get?" We see at the bottom of Fig. 3 a blowup of the upper right of our H-R diagram, now with lines of constant radii marked. The largest stars known in the Milky Way have radii of $1500 \mathrm{R}_{\odot}$, or about 7.2 AU. If you were to take one of these behemoths, and plunk it down where the sun is, its surface would extend to between the orbits of Jupiter and Saturn. Of course, "real" RSGs are known to have highly asymmetrical and messy "surfaces", as witness the high angular resolution images obtained of Betelgeuse by Young et al. (2000).

\section{RSGs in the Magellanic Clouds: Weirder and Weirder}

We naturally wanted to extend this work to RSGs in the Magellanic Clouds, where the metallicities are lower than in the Milky Way. Since the metallicity is lower, we expect that we will need cooler temperatures in order to form the same strength of TiO, the basis for the classification of mid-K through M stars. And, indeed that's just what we found (Paper II): M-type stars are $50 \mathrm{~K}$ and $150 \mathrm{~K}$ cooler in the LMC and SMC, respectively, 


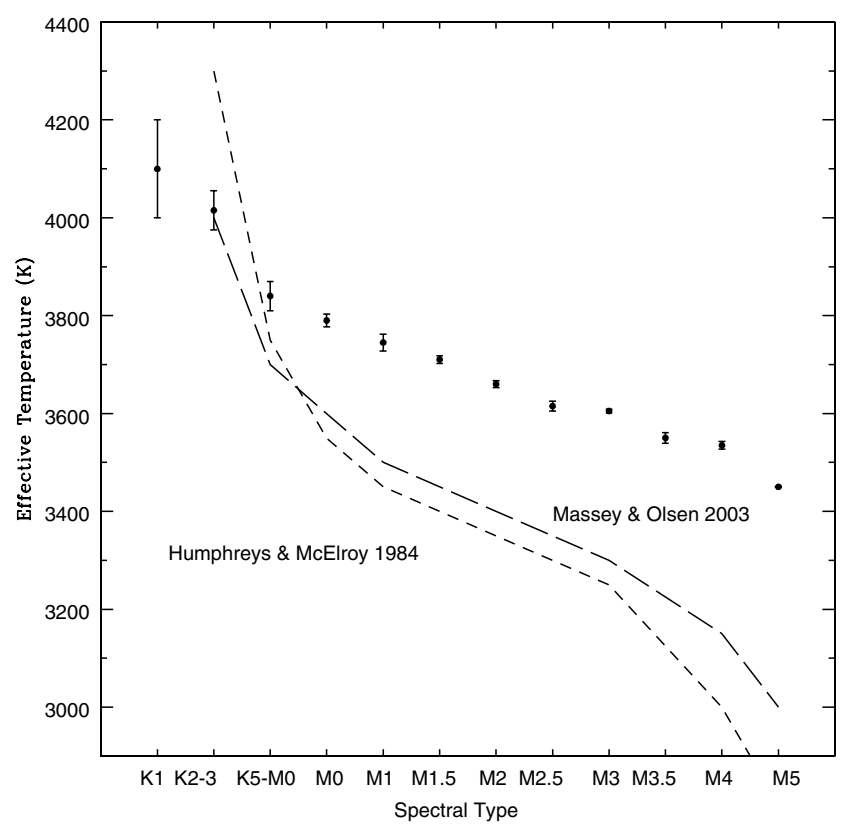

Figure 2. Effective temperature scale for Galactic RSGs. The new temperature scale for RSGs is shown by the points. For comparison, we include the much cooler effective temperatures of Humphreys \& McElroy (1984) and Massey \& Olsen (2003).

compared to their counterparts in the Milky Way. Just as we had for Galactic RSGs, we found great improvement between the observations and the models. For the LMC there is excellent agreement (not shown here; see Fig. 8 in Paper II). For the SMC the results were also improved (Fig. 4), but there were a substantial number of stars that were a bit cooler than the tracks allow.

This "no star zone" beyond the end of the tracks is known as the Hayashi forbidden region - stars in this region are no longer in hydrostatic equilibrium. They shouldn't exist. Even before we had these results, we were intrigued by the fact that there were some stars in the LMC and SMC that were classified as significantly later than the average type by Massey \& Olsen (2003).

However, the real revelation came in our efforts to obtain a spectrum of HV 11423, one of the brightest RSGs in the SMC. It was on our observing list because it was classified as an M0 I by Elias, Frogel, \& Humphreys (1985) (based upon photographic spectra obtained in 1978 and 1979), and we were tired of all of the K-types we had been observing. But, when we took spectra of it in early December 2004 it appeared to be of early K-type, probably K0-1 I. We honestly didn't think much about this at the time, but imagined that perhaps we had gotten the wrong star, although the two spectra we had obtained (on different nights) had matched. The next year (December 2005) we tried again, and took a couple of spectra. Much to our amazement the star was M4 I, much, much later than the spectral types seen for RSGs in the low metallicity SMC. We then went back and checked, and of course we had taken the spectrum of the correct star in 2004 as well - the coordinates left no doubt of that. We took another spectrum the following year, in September (2006), and the star was again an early K. Some digging in various data archives unearthed a VLT/UVES spectrum (apparently never published) 

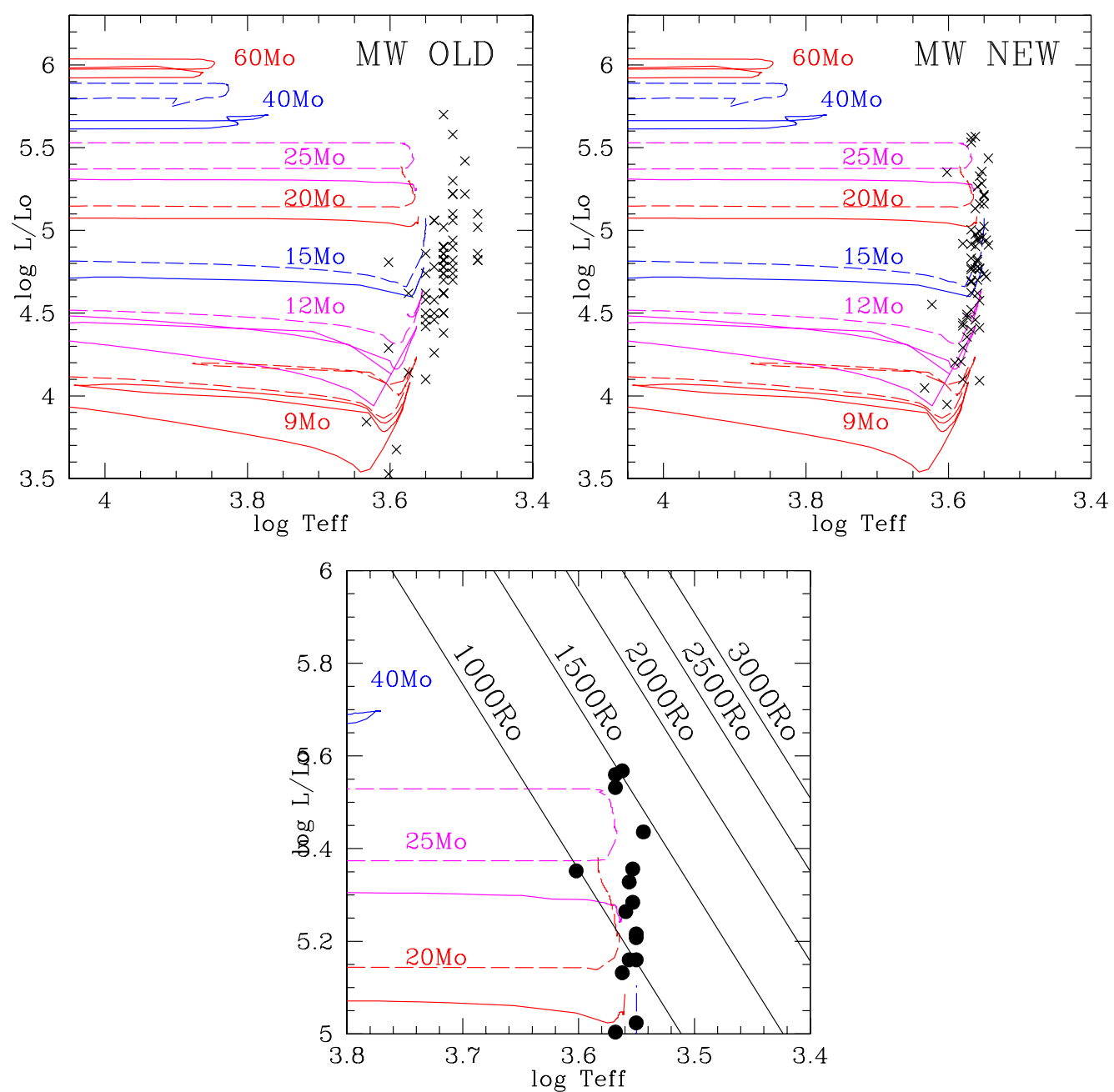

Figure 3. Agreement with evolutionary tracks for the Milky Way. On the left (top) we show the agreement (or rather lack thereof) between the evolutionary tracks and the "observed" location of RSGs using the old effective temperature scale and bolometric corrections given by Humphreys \& McElroy (1984). On the right (top), we show the agreement using the results from Paper I. The evolutionary tracks are the same as those shown for $z=0.020$ in Fig. 1 In the bottom figure we show an expansion of the upper-right part of the later figure, now with lines of constant radii indicated.

taken in December 2001; the star was clearly of even later type than our December 2005 M4 I type - more like an M4.5-5 I. So, here is one of the brightest RSGs in the SMC, and it is doing this funny little jig in the H-R diagram, changing effective temperatures from 3300 to $4300 \mathrm{~K}$ on the time scale of months and no one had noticed. Furthermore, the amount of visual extinction $\left(A_{V}\right)$ changed by more than $1 \mathrm{mag}$, which we attribute to episodic dust formation (see below). Details can be found in Massey et al. (2007).

We concluded that this star is in an unstable period, maybe near the end of its life. Of course, one star is an oddity. The wonderful thing is that Leveque et al. (2007) found several more just like it! We think this underscores just exactly how lightly we've scratched the surface of stellar population studies of even the nearest galaxies. 

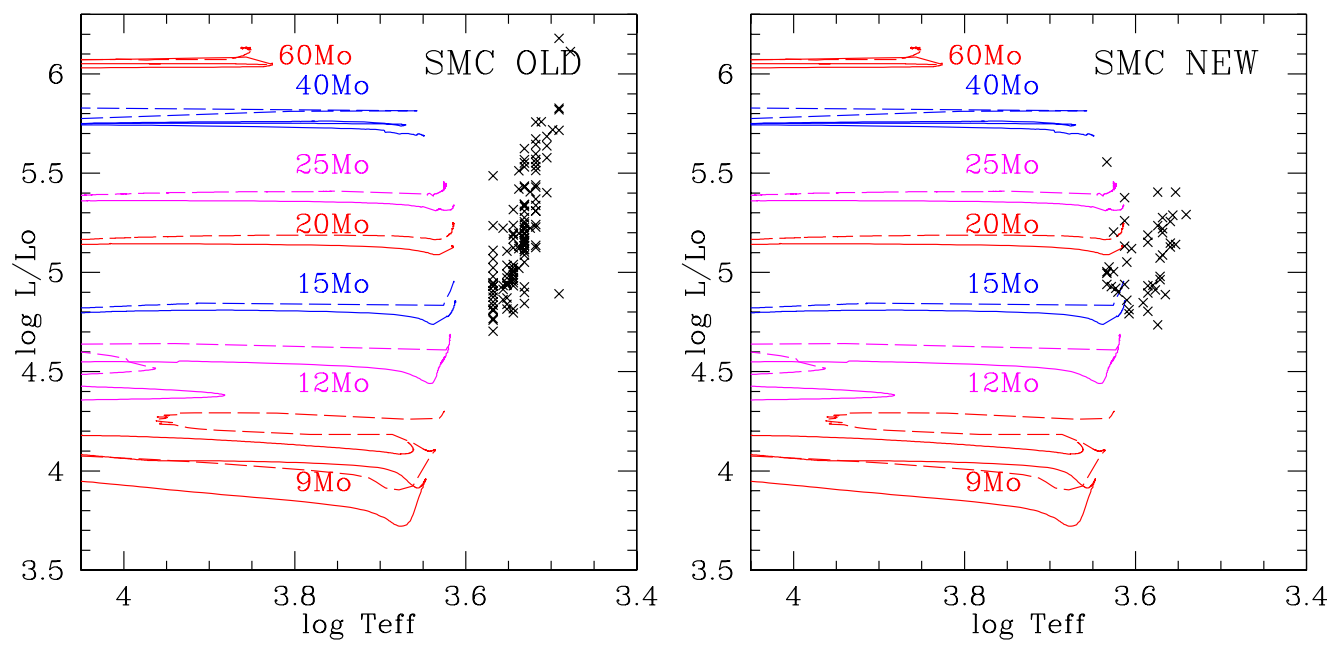

Figure 4. Agreement with evolutionary tracks for the SMC. On the left we show the lack there of)between the evolutionary tracks and the "observed" location of RSGs. On the right, we show the agreement using the results from Paper II. The evolutionary tracks are the same as those shown for $z=0.004$ in Fig. 1.

\section{Self-Consistency: Broad-band colors and VY CMa}

If we only talked about our successes, we would be doing public relations and not science. One of the critical tests we performed was to see if our spectral fitting gave results that were consistent with what we would get from the models if we were to use the broad-band colors $(V-K)_{0}$ and $(V-R)_{0}$ instead. Such a test is not completely independent from our spectral fitting, as we must deredden the broad-band colors to make these comparisons, and for this we adopt the reddenings determined from the spectral fittings, but that is relatively minor. In Fig. 5 (left) we show the comparison between the derived effective temperatures from the spectral fittings, and that obtained from the $(V-K)_{0}$ colors. We see there is a systematic difference that is apparently metallicity-dependent: the median difference is $60 \mathrm{~K}$ for the Milky Way, $105 \mathrm{~K}$ for the $\mathrm{LMC}$, and $170 \mathrm{~K}$ for the SMC, all in the sense the the effective temperatures derived from $(V-K)_{0}$ are hotter. To make the SMC data conform we would have to finagle the $(V-K)_{0}$ calibration by nearly $0.5 \mathrm{mag}$, so this is not due to some sort of subtle photometric transformation issue from $K_{s}$ to $K$ or something. Our first thought was that this was some sort of discrepancy having to do between the fluxes derived from the models and the strengths of the spectral features - the $(V-K)_{0}$ effective temperatures depend upon the former, while the spectral fitting effective temperatures depend upon the latter-but this notion was dispelled by looking at the results from $(V-R)_{0}$. Here we find very good agreement between the effectives temperatures derived from spectral fitting and those from photometry.

Instead, we now believe this is a discrepancy between the effective temperatures derived from the optical and those derived from the near-IR, and may just be due to the intrinsic limitations of static, 1-D models. We know that these stars likely contain cool and warm regions on their surfaces (Freytag, Steffen, \& Dorch 2002), so it would not be unreasonable if the effective temperature one measures is wavelength dependent. This issue is discussed in greater depth in Paper II. 

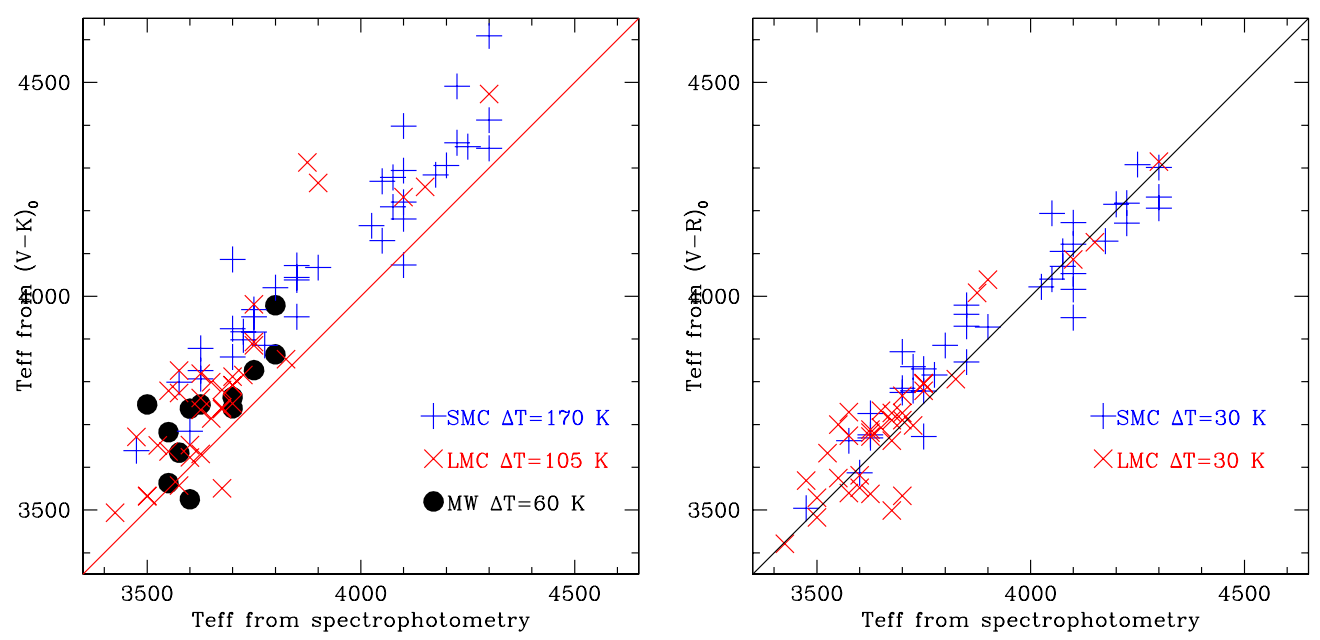

Figure 5. The effective temperatures derived from broad-band photometry are compared to those determined from spectral fitting.

Let us now turn briefly to an analysis we did of VY CMa (Massey, Levesque, \& Plez 2006), where we got both the right and wrong answers. VY CMa is a Galactic RSG with some extreme properties claimed for it in the literature: a luminosity of 2 to $5 \times 10^{5} \mathrm{~L}_{\odot}$, a mass-loss rate of $2 \times 10^{-4} \mathrm{M}_{\odot} \mathrm{yr}^{-1}$, with an effective temperature usually quoted as 2800-3000 K. We were disturbed by these values, as if you plotted the star in the H-R diagram based on these, it would lie well into the Hayashi zone. Yet, the star is fairly stable - George Wallerstein has been observing it spectroscopically for many decades, and the only changes observed have to do with weak emission that originates in the extensive nebulosity around the star. We analyzed the star based upon new optical spectrophotometry and existing optical and $J H K$ photometry, and concluded that the star had an effective temperature of $3650 \mathrm{~K}$ and a luminosity of $0.6 \times 10^{5} \mathrm{~L}_{\odot}$.

There was only one itsy-bitsy problem with these results: they had to be wrong. Once our paper appeared, several colleagues called our attention to the fact that the luminosity we derived for the star was inconsistent with the total luminosity of the system (star plus dust). We should have realized there was a problem ourselves, as we had derived an effective temperature and radius for the surrounding dust shell. The corresponding luminosity of the dust (which we did not work out) is $2.3 \times 10^{5} \mathrm{~L}_{\odot}$, about $4 \times$ larger than what we got for the star itself. Since the dust is heated by the star, this is impossible.

About the only way we have found out of this would be if there was substantial extra grey extinction. We get $A_{V}$ by reddening the models of appropriate temperature to match the shape of the stellar continuum using a $R_{V}=3.1$ Cardelli, Clayton, \& Mathis (1989) law. However, if the copious dust surrounding VY CMa has a distribution of grain sizes which is skewed towards larger sizes than usual, then we would underestimate $A_{V}$ as the dust would be greyer than we assume. We would need about an additional 1.5-2.0 mag of such grey extinction. In any event, if we take our effective temperature, and a luminosity of 3 or $4 \times 10^{5} \mathrm{~L}_{\odot}$ then the star sits in a very reasonable place in the H-R diagram, near the upper luminosity limit for RSGs.

Still, we don't think this reveals some fundamental flaw with what we're doing. The amount of dust around VY CMa is quite unusual (see Smith et al. 2001), and it will be of interest to determine the properties of this dust. 


\section{When Smoke Gets in Your Eyes}

One of the things that worried us when we were doing our fits was that there were some stars for which there was very poor agreement in the near-UV (i.e., $<4100 \AA$, hereafter NUV), always in the sense that the star had more flux than the best-fitting model. We illustrate an example in Fig. 6 (left) for the star KY Cyg. Now, we considered a number of possibilities. Were these binaries, with the NUV being contributed by a hot companion? We didn't think so. We had indeed found some stars that clearly were binaries-but this was evident by having a composite spectrum, with Balmer lines clearly evident. The remaining stars that showed extra flux in the near-UV didn't exhibit any signs of a composite spectrum. So that didn't wash as an explanation.

We investigated this further in Massey et al. (2005b). Was this due to a problem with the models? We didn't think so, because there were lots of stars that didn't show this problem, and there didn't seem to be any correlation with effective temperature. What the NUV problem did show a correlation with was the amount of visual extinctionstars with the largest NUV problem also had the largest $A_{V}$. We looked into this a little more deeply, and indeed it turned out that the stars with the largest $A_{V}$ actually had a considerable amount of excess extinction compared to OB stars in the same OB associations. This is illustrated in Fig. 6. The error bars show the typical sigma of $A_{V}$ of the OB stars in a given association. For most RSGs there was good correlation between the $A_{V}$ found for the OB stars, and the $A_{V}$ found for the RSGs, but for some significant fraction of the RSGs there was significantly more extinction - up to several magnitudest. We see the same thing for RSGs in the Magellanic Clouds: in both the SMC and LMC RSGs show greater extinction than the OB stars (Paper II).

If the extra extinction was due to circumstellar dust, then that could also explain the extra flux in the NUV - light near the star would be scattered by the dust, making the light more blue. (This is different than the effect of dust along the line of sight.) But, no one had suggested that the observed dust mass-loss rates would lead to significant circumstellar extinction around RSGs. We did the math, though (Massey et al. 2005b),

$\dagger$ The alert reader may realize that $A_{V}$ determined by $E(B-V)$ from broad-band colors requires a different effective $R_{V}^{\prime}$ for intrinsically red stars than for intrinsically blue stars. We derive our $A_{V}$ from spectrophotometry, so this issue doesn't apply. Still, if you are trying to do this for RSGs from broad-band photometry, then $R_{V}^{\prime}=4.1+0.1 E(B-V)-0.2 \log g$; see discussion in Massey et al. (2005b).
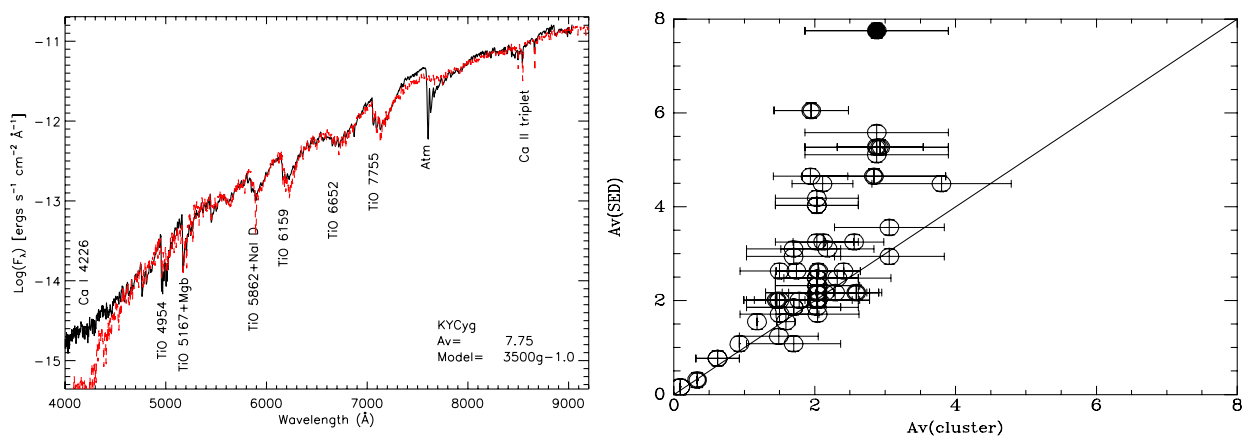

Figure 6. The effects of circumstellar dust? On the left we show the NUV problem for the star KY Cyg. Note that the star (solid line) has far more flux in the NUV than does the reddened model (dashed). On the right the RSG extinction is plotted against that of the OB stars in the same associations. The error bars denote the range of extinction of the OB stars. The filled circle at the top denotes KY Cyg, which has one of the worst NUV problems. 
and this really was exactly what you would expect: a thin-shell approximation (10 yr of dust mass loss at a rate of $10^{-8} \mathrm{M}_{\odot} \mathrm{yr}^{-1}$ condensing out at distance of ten stellar radii) should lead to $>1$ mag of visual extinction.

Exactly how much dust do RSGs contribute to the ISM? In our work, we found a nice correlation between the bolometric luminosity of a star and its dust mass-loss rate. With this we were then able to estimate the amount of dust that RSGs contribute locally, about $3 \times 10^{-8} \mathrm{M}_{\odot} \mathrm{yr}^{-1} \mathrm{kpc}^{-2}$, in good agreement with the value estimated by Jura \& Kleinmann (1990). This is probably $3 \times$ less than that contributed by late-type WCs in the solar neighborhood, and about $200 \times$ less than that contributed by asymptotic giant branch (AGB) stars. So, locally, they don't amount to much as dust producers. However, in a metal-poor starburst, or in galaxies at large look-back time, one would expect RSGs to dominate the production of dust, as late-type WCs are not found in metal-poor systems, and AGBs require several Gyr to form.

Before leaving this subject, I'd like to briefly address the subject of RSG mass-loss. We don't know what drives the mass-loss of RSGs: arguments have been presented both for pulsation and for having the dust drive the wind. But, I'd like to quote something my colleague Stan Owocki wrote in an email about all this, contrasting RSG mass-loss with $\mathrm{O}$ star mass-loss. The escape velocity from a star is just $620 \mathrm{~km} \mathrm{~s}^{-1} \times \sqrt{(M / R)}$. $\mathrm{O}$ stars have a $\mathrm{M} / \mathrm{R}$ ratio that is of order unity, but not RSGs! There the ratio is much smaller, more like 0.02 . So, the escape velocity is down by a factor of 7 or so, under $100 \mathrm{~km} \mathrm{~s}^{-1}$. Stan argued that the mass-loss of a hot star is set by conditions outside the stellar interior, i.e., opacity in the atmosphere and wind, that results in the classic CAK mass loss (Castor, Abbott, \& Klein 1975). A RSG, on the other hand, suffers mass loss because the "heavy lifting" has been done by the interior, as a significant fraction of the luminosity of the star has gone into making a bigger radius. So, Stan argues, it is kind of like walking with a nearly full glass of water (RSG) vs a glass that is only $1 \%$ full (O star) - even a small jiggle can lead to big changes in the mass loss for a RSG.

\section{The Future}

Where do we go from here? Our group is working on several projects. One of this is to extend these studies to other metal-poor galaxies, particularly WLM, and see if (for instance) we can find more wacky late-type RSGs like HV 11423 and its friends. Another is to extend this to M31, where the metallicity is $2 \times$ solar, at least according to studies of nebular abundances (for instance, Zaritsky, Kennicutt, \& Huchra 1994). This brings us to one of our preliminary results. Abundance studies of several M31 A- and B-type supergiants by Venn et al. (2000) and Smartt et al. (2001) found abundances that were essentially solar, not $2 \times$ solar. This is of course confusing, as it flies in the face of everything we know (or thought we knew!) about one of our nearest neighboring galaxies.

We can comment on this briefly. The observed upper luminosity of the RSGs is consistent with that expected based on the $2 \times$ solar tracks but not the solar metallicity tracks. This is illustrated in Fig. 7. If the metallicity were truly solar, then where are of the high luminosity RSGs? The ones that would come from $25 \mathrm{M}_{\odot}$ ? (Compare also to Fig. 3, upper right.) But the $2 \times$ solar models work very well. I was gratified to learn at this conference that Norbert Przybilla finds similarly high abundances for these Atype supergiants in his reanalysis, using the improved photometry that our Local Group Galaxies Survey has provided. 

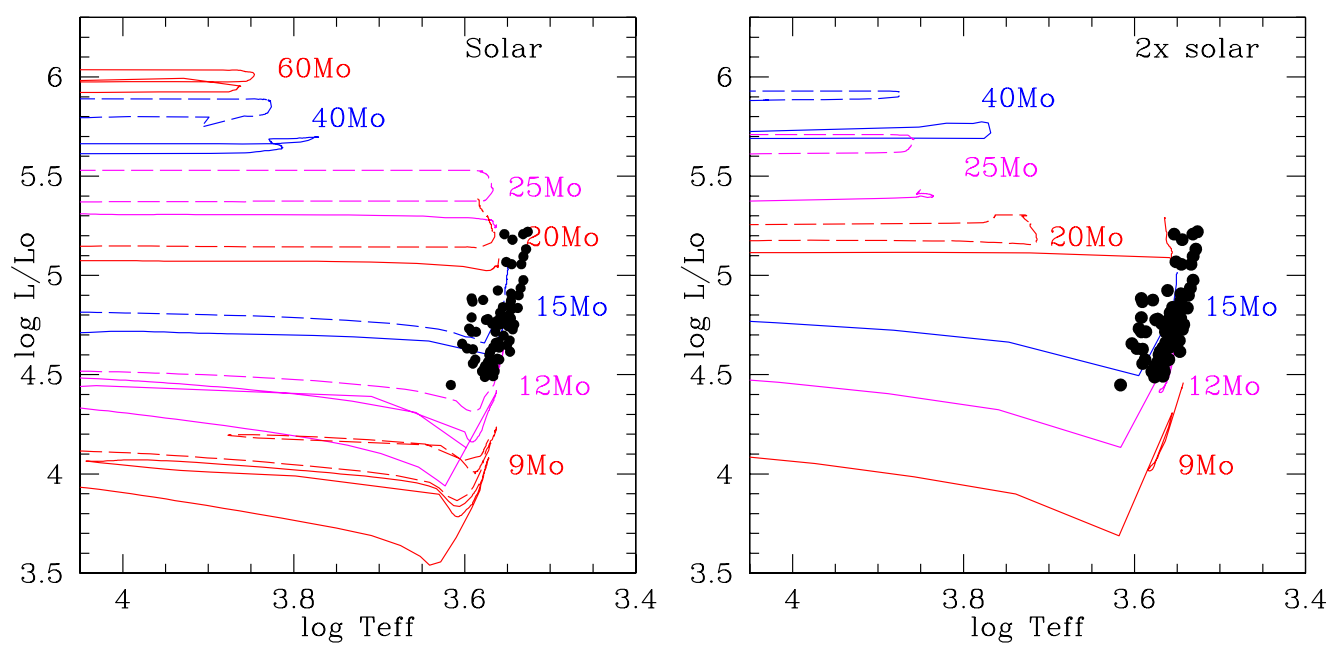

Figure 7. RSGs in M31 compared to solar (left) and $2 \times$ solar (right) tracks. The evolutionary tracks used are the same as in Fig. 1. The distribution works well for the $2 \times$ tracks, but not the solar tracks, which predict higher luminosity RSGs than what are observed.

\section{Acknowledgements}

We thank our colleagues Georges Meynet and Andre Maeder, who co-authored several of the papers we discussed here and who have always been very generous by making their work available to others. Geoff Clayton and David Silva are also working with us. This work is partially supported through the National Science Foundation (AST-0604569).

\section{References}

Abbott, D. C. \& Hummer, D. G. 1985, ApJ, 294, 286

Auer, L. H. \& Mihalas, D. 1972, ApJS, 24, 193

Cardelli, J. A., Clayton, G. C., \& Mathis, J. S. 1989, ApJ, 345, 245

Castor, J. I., Abbott, D. C., \& Klein, R. I. 1975, ApJ, 195, 157

Conti, P. S. 1973, ApJ, 179, 181

Crowther, P. A. 2007, ARAESA, 45, 177

Elias, J. H., Frogel, J. A., \& Humphreys, R. M. 1985, ApJS, 57, 91

Freytag, B., Steffen, M., \& Dorch, B. 2002, Astron. Nach., 323, 213

Gabler, R., Gabler, A., Kudritzki, R. P., et al. 1989, A\&A, 226, 162

Garmany, C. D. \& Stencil, R. E. 1992, A\&SAS, 94, 211

Gustafsson, B., Bell, R. A., Eriksson, K., \& Nordlund, A.. 1975, A\& A, 42, 407

Gustafsson, B., Edvardsson, B., Eriksson, et al. 2003, in: I. Hubeny, D. Mihalas, \& K. Werner (eds.), Stellar Atmosphere Modeling (San Francisco: ASP), ASP Conf. Ser., 288, 331

Herrero, A., Puls, J., \& Najarro, F. 2002, A\&SA, 396, 949

Hillier, D., Lanz, T., Heap, S. R., et al. 2003, ApJ, 588, 1039

Hillier, D. \& Miller, D. L. 1989, ApJ, 496, 407

Humphreys, R. M. 1978, ApJS, 38, 309

Humphreys, R. M. 1979a, ApS, 39, 389

Humphreys, R. M. 1979b, ApJ, 345, 854

Humphreys, R. M. 1980a, ApJ, 238, 65

Humphreys, R. M. 1980b, ApJ, 241, 587

Humphreys, R. M. 1980c, ApJ, 241, 598

Humphreys, R. M. \& Davidson, K. 1979, ApJ, 232, 409

Humphreys, R. M. \& McElroy, D. B. 1984, ApJ, 284, 565 
Humphreys, R. M. \& Sandage, A. 1980, ApJS, 44, 319

Jura, M. \& Kleinmann, S. G. 1990, ApJS, 73, 769

Kudritzki, R. P. 1976, A\& $A, 552,11$

Kudritzki, R. P. \& Hummer, D. G. 1990, ARA $\mathscr{E} A$, 28, 303

Lamers, H. J. G. L. M. 1997, in: A. Nota \& H. J. G. L. M. Lamers (eds.), Luminous Blue Variables: Massive Stars in Transition, (San Francisco: ASP), ASP Conf. Ser, 120, 76

Levesque, E. M., Massey, P., Olsen, K. A. G., et al. 2005, ApJ, 628, 973

Levesque, E. M., Massey, P., Olsen, K. A. G., et al.. 2006, ApJ, 645, 1102

Levesque, E. M., Massey, P., Olsen, K. A. G., \& Plez, B. 2007, ApJ, 667, 202

Maeder, A. \& Meynet, G. 1987, A\&3A, 182, 243

Massey, P. 2003a, ARAEA, 41, 15

Massey, P. 2003b, in: K. A. van der Hucht, A. Herrero \& C. Esteban (eds.), A Massive Star Odyssey, from Main Sequence to Supernova (San Francisco: ASP), Proc. IAU Symp. 212, 316

Massey, P., Bresolin, F., Kudritzki, R. P., et al. 2004, ApJ, 608, 1001

Massey, P., Levesque, E. M., Olsen, K. A. G., et al. 2007, ApJ, 660, 301

Massey, P., Levesque, E. M., \& Plez, B. 2006, ApJ, 646, 1203

Massey, P., \& Olsen, K. A. G. 2003, AJ, 126, 2867

Massey, P., Plez, B., Levesque, E. M., et al. 2005b, ApJ, 634, 1286

Massey, P., Puls, J., Pauldrach, A. W. A., et al. 2005a, ApJ, 627, 477

Meynet, G. \& Maeder, A. 2000, $A \mathscr{E} A, 361,101$

Meynet, G. \& Maeder, A. 2003, $A \mathscr{E} A, 404,975$

Meynet, G. \& Maeder, A. 2005, A\&SA, 429, 581

Meynet, G., Maeder, A., Schaller, G., et al. 1994, A\&BA, 103, 97

Mihalas, D. \& Auer, L. H. 1970, ApJ, 160, 1161

Plez, B. 2003, in: U. Munari (ed.), GAIA Spectroscopy: Science and Technology (San Francisco: ASP), ASP Conf. Ser., 298, 189

Plez, B., Brett, J. M., \& Nordlund, Å. 1992, AษA, 256, 551

Puls, J., Kudritzki, R. P., Herrero, A., et al. 1996, A\&A, 305, 171

Smartt, S. J., Crowther, P. A., Dufton, P. L., et al. 2001, MNRAS, 325, 257

Smith, N., Humphreys, R. M., Davidson, K., et al. 2001, AJ, 121, 1111

Smith, N. \& Owocki, S. P. 2006, ApJ, 645, 45

Venn, K. A., McCarthy, J. K., Lennon, D. J., et al. 2000, ApJ, 541, 610

Young, J. S., Baldwin, J. E., Boysen, R. C., et al. 2000, MNRAS, 315, 635

Zaritsky, D., Kennicutt, R. C., \& Huchra, J. P. 1994, ApJ, 42087

\section{Discussion}

Kudritzki: You use the TiO molecular lines as a $T_{\text {eff }}$ diagnostic. This depends on an assumption about the oxygen abundances. Which did you use for the Milky Way? And then for LMC, SMC?

MAsseY: Relative to the Sun, we used $\log Z=0.0$ for the Milky Way, $\log Z=-0.3$ for the LMC, and $\log Z=-0.75$ for the SMC, based primarily on the nebular oxygen abundances we know for the latter two. But, the oxygen abundance is actually irrelevant. The amount of $\mathrm{TiO}$ you get is controlled by the amount of $\mathrm{Ti}$, not $\mathrm{O}$, because $\mathrm{Ti}$ is so much rarer than O. There's good discussion of this point in Plez (2003).

PRZYBILlA: From the analysis of a couple of A-type supergiants in M31, we derive oversolar abundances, about 30-40\% above the Grevesse \& Sauval (1998, Spa. Sci. Rev. 85, 161) values.

MASSEY: Good! When I refer to ' 2 x solar' I mean $\log \mathrm{O} / \mathrm{H}+12=9.0$ if I'm talking about the nebular abundances, or $Z=0.040$ if I'm talking about the available evolutionary 
models. If I understood you correctly, you've revised the abundances of the Venn et al. A-type supergiants to $\log \mathrm{O} / \mathrm{H}+12=9$ due to improved estimates of the luminosities. So, maybe the agreement about the metallicity is better than I thought now.

Moffat: Exactly a year ago in Carilo celebrating Virpi's 70th birthday, I put you on the spot. Now it is a year later, so may I ask you the same question? Well, open cluster Westerlund 1 has a large number of RSGs yet its turn-off mass is close to $40 \mathrm{M}_{\odot}$, not $25 \mathrm{M}_{\odot}$. This would then be an exception to your findings that RSGs come from stars of mass $10 \mathrm{M}_{\odot}<M_{i}<25 \mathrm{M}_{\odot}$. Do you have an explanation for this?

MASSEY: It would work if the cluster is not strictly coeval.

Crowther: One might reconcile the existence of RSGs in Westerlund $1\left(40 \mathrm{M}_{\odot}\right.$ turn off) with Geneva rotating models at $\mathrm{Z}_{\odot}$ by adopting reduced initial rotation velocities (100 $\mathrm{km} \mathrm{s}^{-1}$ cf. $300 \mathrm{~km} \mathrm{~s}^{-1}$ generally adopted). There is no evidence for age differences between RSGs and other massive stars in Westerlund 1.

LEITHERER: Did you include in your sample stars with good Hipparcos distances and/or diameter measurements like $\alpha$ Her, $\alpha$ Sco, or $\alpha$ Ori? That should give you an additional handle on $T_{\text {eff }}$.

MASSEY: We restricted our sample to stars in OB associations, both for the distances and because (naively) I thought we might need to know the typical reddenings from the OB stars. But we did include a few famous RSGs as spectral standards, such as $\alpha$ Her.

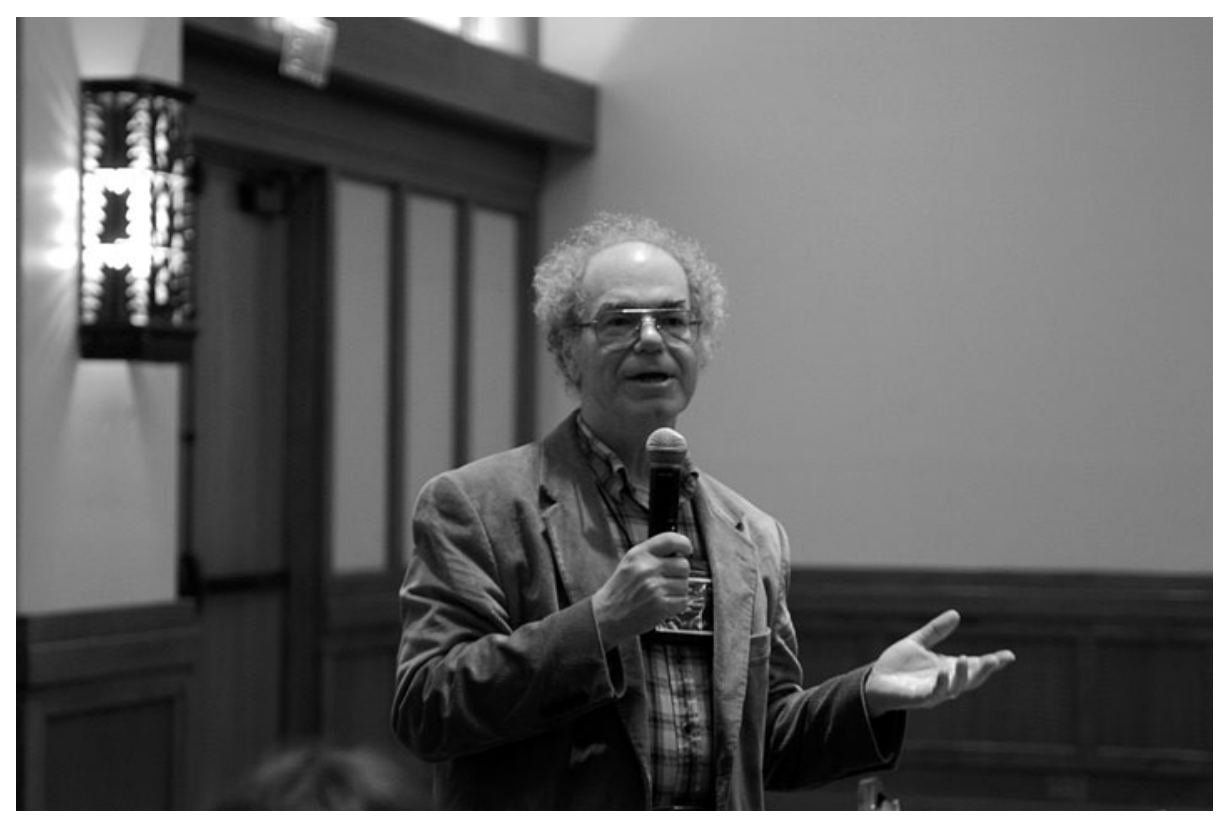

Phil Massey. 


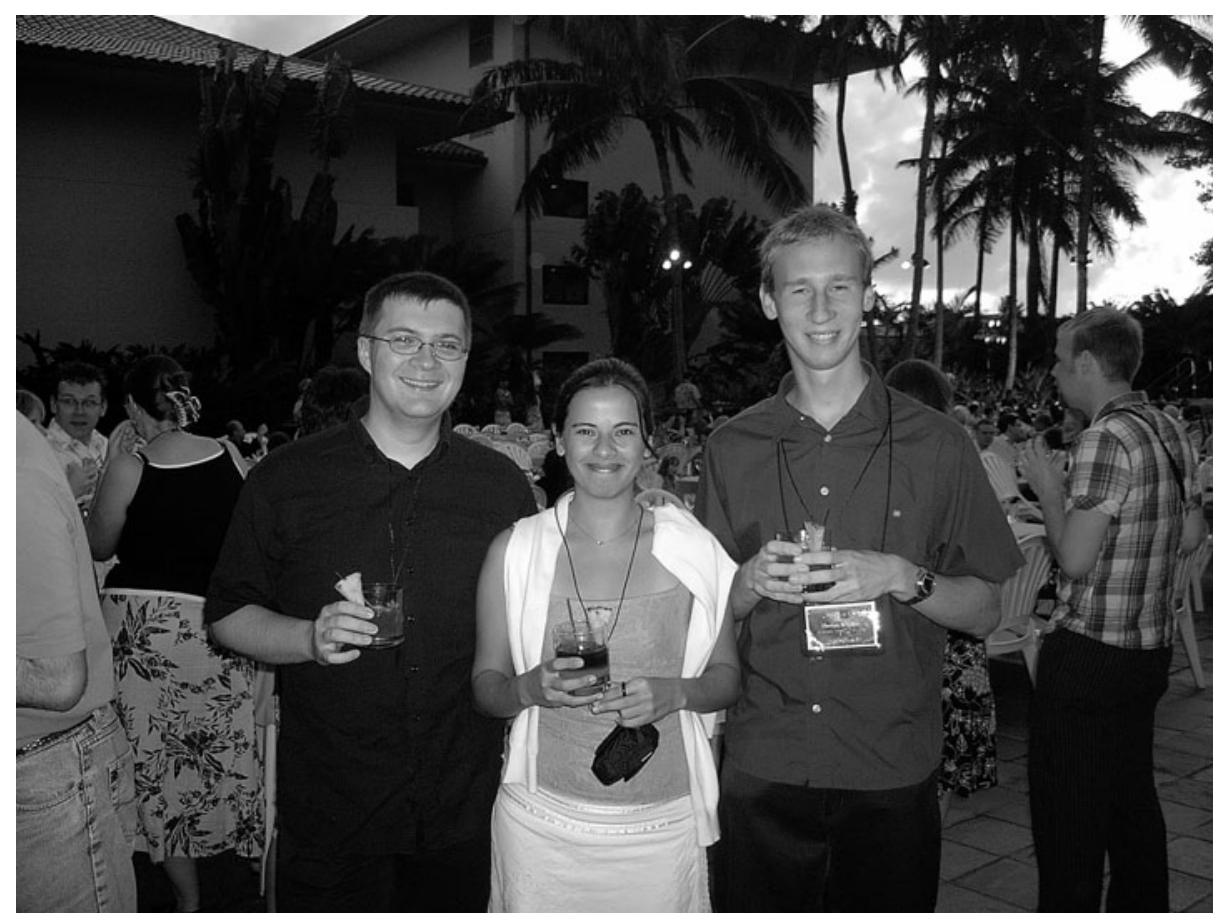

Norbert Przybilla, Maria Nieva and Florian Schiller.

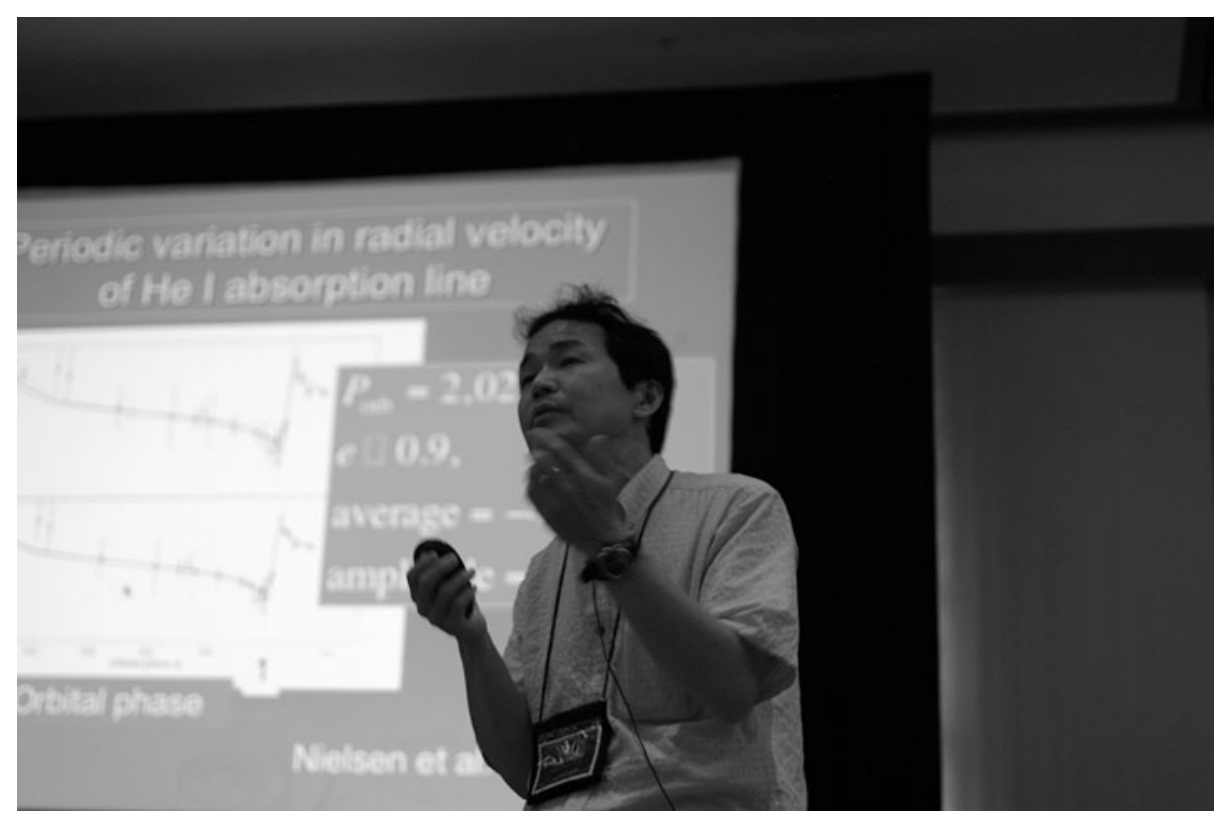

Atsuo Okazaki. 